\title{
IMPROVED METHOD FOR ORBITAL ELEMENTS DIFFERENTIAL CORRECTION.
}

\author{
A. López García ${ }^{1)}$, J. A. López Orti1)2), R. López Machíi ${ }^{1) 2)}$. \\ 1) Observatorio Astronómico de la Universidad de Valencia. \\ 1) Departamento de Matemática Aplicada y Astronomía. \\ 2) Colegio Universitario de Castellón. \\ Universidad de Valencia.
}

\begin{abstract}
As was pointed out by IAU in 1976, an important problem in fundamental astronomy is the improvement of the vernal equinox and equator positions. To this aim, it is necessary to know the accurate values of minor planets orbital elements. The classical methods of orbital elements differential corrections are based on linking the observations at different epochs considering equal derivatives respect to the initial and osculating elements.

In this paper we present an improved method in which the least squares matrix coefficients is calculated from the integration of the Lagrange planetary equations and its derivatives.

\section{INTRODUCTION}

One of the fundamental problems in positional astronomy is the determination of the equator and the vernal equinox. This problem has been faced traditionally by meridian observations of the Sun and the inner planets. Actually, other alternative methods are used to this aim, as the ocultations of stars by the Moon and the programs of astrometry of asteroids (coordinated by the ITA of Leningrad).

In this way, as a natural continuation of the method developed to the improvement of minor planets orbital elements (Lopez García et al.; 1989), we have studied its inclusion in the method of vernal equinox correction (Calaf, J.; Catalá, Mª .A.; 1983).

As it is known the Minor Planets elements change in time due to planetary perturbations and it is no possible, strictly speaking, to apply differential corrections taking these elements as constants, mainly when the observations are far in time.

The main limitation which arises in the mean least-squares classical methods to the differential elements correction is the difficulty of to replace the partial derivatives of the O-C residual, respect to the epoch elements by its derivatives respect to the initial epoch elements, to be able to obtain the correction of these.

In the present work we have completed our orbital elements correction algorithm, including the corrections to the catalog equinox. This algorithm allows to use ephemeris far in time, linking them by means of the integration of the planetary equations of Lagrange and its derivatives. We use the planetary theory VSOP87 (Bretagnon, P.; Francou, G., 1988), and we finally get a linear system to improve the initial elements for the epoch $t_{0}$ and the vernal equinox of the reference used catalog.
\end{abstract}




\section{FORMATION OF NORMAL EQUATIONS.}

The starting point is to consider a set of $N$ asteroids and to have $n_{r}(r=1, \ldots, N)$ observations of each one. Taking $\overrightarrow{\sigma_{r}^{0}}$ the orbital elements of a minor planet $r$ for the epoch $t_{0}$, $\left(\alpha_{i}^{r}, \delta_{i}^{r}\right)$ the observed positions for other $t^{r}$ epoch, $\alpha\left(\overrightarrow{\sigma_{r}^{0}}, t_{i}^{r}\right), \delta\left(\overrightarrow{\sigma_{r}^{0}}, t_{i}^{r}\right)$ the positions for $t_{i}^{r}$, integrated from the elements $\overrightarrow{\sigma_{\mathrm{r}}^{0}}$, and $\Delta \xi, \Delta \eta$ the errors in RA and Dec, respectively, for the vernal equinox in the used catalog, for the epoch $t_{0}$ the true elements will be $\overrightarrow{\sigma_{r}^{0}}+\Delta \overrightarrow{\sigma_{r}^{0}}$, which will be determinated by the condition of minimum of

$$
\mathrm{R}\left(\Delta \overrightarrow{\sigma_{1}^{0}}, \ldots, \overrightarrow{\Delta \sigma_{N}^{0}}, \Delta \xi, \Delta \eta\right)=\sum_{\mathrm{r}=1}^{\mathrm{N}} \sum_{\mathrm{i}=1}^{\mathrm{n}_{\mathrm{r}}}\left[\left(\Delta \alpha_{\mathrm{i}}^{\mathrm{r}}\right)^{2} \cdot \cos ^{2} \delta_{\mathrm{i}}^{\mathrm{r}}+\left(\Delta \delta_{\mathrm{i}}^{\mathrm{r}}\right)^{2}\right]
$$

where

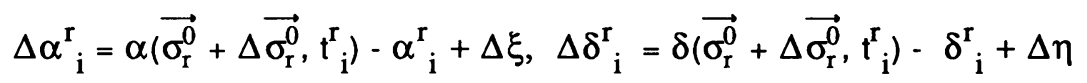

The condition of minimum in Eq. 1 can be obtained in a linear form for $\Delta \overrightarrow{\sigma_{\mathrm{r}}^{0}}, \Delta \xi$, and $\Delta \eta$, by means of the integration of Lagrange's planetary equations and its derivatives. For this purpose we may use the technique shown in (Simon, J.L.; 1987).

With this we finally get a $6 \mathrm{~N}+2$ linear system equations.

The solution of this linear system provides the initial elements correction $\Delta \overrightarrow{\sigma_{\mathrm{r}}^{0}}$, so as the corrections $\Delta \xi$ and $\Delta \eta$. The integration of the Lagrange's equations from $t_{0}$ to $t$ taking $\overrightarrow{\sigma_{\mathrm{r}}^{0}}+\Delta \overrightarrow{\sigma_{\mathrm{r}}^{0}}$ as initial values gives the corrected elements $\overrightarrow{\sigma_{\mathrm{r}}}$ for the desired osculating epoch.

\section{CONCLUSIONS}

In the present paper we develop an algorithm for the correction of minor planet elements varying with time, that allows to link all the observations available for each minor planet in an only adjust that also includes the vernal equinox corrections. The main advantage of this algorithm is that allows to calculate the derivatives of the residuals in $\alpha$ and $\delta$ respect to the elements of the initial epoch, instead of respect to the osculating elements.

\section{REFERENCES}

Bretagnon, P.; Francou, G. Astron. Astrophys., 202, 309-315. (1988).

Brower, D.; Clemmence, G. M. METHODS OF CELESTIAL MECHANICS. Academic Press. (1961).

Calaf, J.; Catalá, Ma.a.A., Actas IV Asamblea Nacional de Astronomía y Astrofísica. Vol. II, 941-954. Santiago, (1983).

López García et al. Asteroids, Comets, Meteors III. Com. nº 160. Uppsala, (1989).

Simon, J. L. Astron. Astrophys., 175, 303-308. (1987). 